University of Nebraska - Lincoln

DigitalCommons@University of Nebraska - Lincoln

Public Health Resources

Public Health Resources

2002

\title{
Epidemiological Measures of Risk of Malaria
}

J. Kevin Baird

ALERTAsia Foundation, jkevinbaird@yahoo.com

Michael J. Bangs

Kasetsart University

Jason D. Maguire

U.S. Navy

Mazie J. Barcus

U.S. Navy

Follow this and additional works at: https://digitalcommons.unl.edu/publichealthresources

Baird, J. Kevin; Bangs, Michael J.; Maguire, Jason D.; and Barcus, Mazie J., "Epidemiological Measures of Risk of Malaria" (2002). Public Health Resources. 385.

https://digitalcommons.unl.edu/publichealthresources/385

This Article is brought to you for free and open access by the Public Health Resources at DigitalCommons@University of Nebraska - Lincoln. It has been accepted for inclusion in Public Health Resources by an authorized administrator of DigitalCommons@University of Nebraska - Lincoln. 


\title{
Epidemiological Measures of Risk of Malaria
}

\author{
J. Kevin Baird, Michael J. Bangs, Jason D. Maguire, and Mazie J. Barcus
}

\section{Introduction}

Estimates of the risk of infection by the parasites that cause malaria govern decisions regarding vector control, chemoprophylaxis, therapeutic management, and clinical classifications of immunological susceptibility to infection. Gauging the risk of malaria represents a critical step in its management and the investigation of its consequences. The term malariometry is applied to the numerical measure of risk of malaria in communities (9). Many approaches have been developed and applied to malariometry, but no single method stands out as universally applicable. Instead, individual measures of risk must be suitable for specific questions posed in the context of what may be practically measured. For example, passive surveillance provides a superior measure of risk where the infrastructure of diagnosis and reporting is well developed and the risk of infection relatively low, e.g., in the United States, where conducting active cross-sectional surveys would yield little useful information at great cost. Active surveillance for cases is suited to areas with relatively high risk, unreliable diagnostic capabilities and inadequate reporting infrastructure. This chapter strives to catalog measures of risk of malaria and define their utility in the context of local parameters of endemicity, infrastructure, and intent of inquiry.

The risk of malaria is highly dependent on interactions between the host, parasite, mosquito vector, and environment, a relationship known as the epidemiologic triad of disease. Changes in any one of these elements may profoundly impact risk of infection. Measures of risk of malaria may be broadly classified as either indirect or direct. Indirect measures gauge risk through surrogate markers of risk of infection such as rainfall, altitude, temperature, entomological parameters, spleen rates, antibody titers, or patterns of antimalarial drug use in a community. Direct measures of risk depend on diagnoses of malaria (clinical or microscopic) and their relationship to a variety of denominators representing classes of persons at risk over some unit of time. In general, indirect measures apply data conveniently at hand to estimate risk of malaria. By contrast, direct estimates of risk often require deliberate effort to collect data for the sole purpose of gauging risk of malaria.

An area supporting active malaria transmission is termed endemic. Transmission of infection may be unstable or stable, the primary difference being a fluctuating low to high incidence versus a consistently high incidence over successive years. Malariologists have long graded endemic malaria according to risk of infection as reflected

From: Methods in Molecular Medicine, Vol. 72: Malaria Methods and Protocols Edited by: Denise L. Doolan (c) Humana Press, Inc., Totowa, NJ 
in the proportion of children and adults having enlarged spleens (spleen rate, see below). However, these terms have evolved into a more general use and are routinely applied in the absence of supporting spleen rate measures. The following terms have been used to empirically gauge regional risk according to criteria described by Bruce-Chwatt (9):

1. Hypoendemic: Little transmission, and the effects of malaria on the community are unimportant.

2. Mesoendemic: Variable transmission that fluctuates with changes in one or many local conditions, e.g., weather or disturbance to the environment.

3. Hyperendemic: Seasonally intense malaria transmission with disease in all age groups.

4. Holoendemic: Perennial intense transmission with protective clinical immunity among adults.

\section{Indirect Estimates of Risk of Malaria}

\subsection{Environmental (Rainfall, Altitude, Temperature)}

Transmission of malaria requires mosquito vectors in the genus Anopheles. These insects exhibit exquisite sensitivity to the environmental parameters of temperature and humidity. Thus, rainfall, altitude, and temperature govern the activity and abundance of anopheline mosquitoes and the transmission of malaria. Within ranges of temperature $\left(20-30^{\circ} \mathrm{C}\right)$ and humidity $(>60 \%)$ that vary for each vector species, the mosquito survives and is capable of transmitting malaria. When the limits of temperature and humidity tolerance are exceeded, the vectors die, and the risk of malaria evaporates. Variations of temperature and humidity within the viable range for mosquitoes can also affect the duration of sporogony, the time required for development of the parasite in the mosquito after taking a bloodmeal from an infected human so that a new host can be infected. Cooler temperatures generally prolong sporogony, decreasing the period of infectivity. High relative humidity increases mosquito life-span, so that each infective mosquito can infect more hosts. The risk of transmission by anophelines, however, depends on an available pool of infectious humans so that even in a favorable environment with the appropriate vector, transmission cannot be sustained without adequate numbers of already infected humans. Conditions perfectly suitable for anopheline survival allow seasonably abundant mosquito populations in the United States, but infection is rare because of the lack of infectious humans in the region.

\subsection{Entomological}

Chapter 1 details the use of the entomological inoculation rate as a measure of risk of infection. The following terms have been used to describe risk of malaria according to entomological criteria:

1. Human landing rate $=$ anophelines captured/person-night.

2. Infected mosquito $=$ oocysts in stomach wall by dissection.

3. Infective mosquito $=$ sporozoites in salivary gland by dissection.

4. Sporozoite rate $=$ infective anophelines/anophelines captured.

5. Entomological inoculation rate $=$ human landing rate $\times$ sporozoite rate. $=$ infective mosquito bites/person-night.

\subsection{Clinical}

\subsubsection{Spleen Rate}

The spleen rate is the proportion of people in a given population having enlarged spleens expressed as a percentage. The relationship between malaria and the spleen 
Table 1

WHO Criteria for Classification of Endemicity by Spleen Rates

\begin{tabular}{lcc}
\hline Endemicity & Children aged 2-9yr $(\%)$ & Adults $(>16 \mathrm{yr})$ \\
\hline Hypoendemic & $0-10$ & No measure \\
Mesoendemic & $11-50$ & No measure \\
Hyperendemic & $>50$ & "High" $(\geq 25 \%)$ \\
Holoendemic & $>75$ & "Low" $(<25 \%)$ \\
\hline
\end{tabular}

\section{Table 2}

\section{Criteria for Scoring Spleen Size ${ }^{a}$}

Score Description of spleen as measured with subject in recumbent position

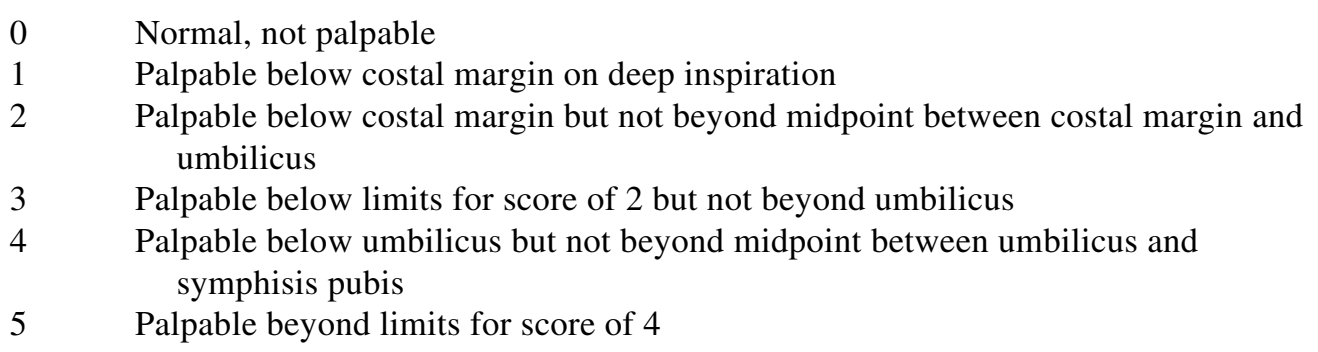

${ }^{a} \mathrm{AES}=\Sigma\left(\right.$ Hackett score $\left._{\mathrm{i}} \times n_{\mathrm{i}}\right) / N$, where Hackett scores of 1 through 5 are included. $n_{\mathrm{i}}=$ number of spleens measured with a given Hackett score. $N=n_{1}+n_{2}+n_{3}+n_{4}+n_{5}$ (total number of non-Hackett 0 spleens measured)

rate has served as an indirect marker of risk of malaria long before plasmodia were known as the cause (Dempster introduced the method in India in 1848). Chronic exposure to malaria causes spleen enlargement. Thus, the percentage of people having enlarged spleens and the degree of enlargement correlate with risk of infection in a community. The WHO has classified endemicity as gauged by spleen rate as shown in Table 1.

Important caveats complicate this convenient estimate of risk. Under conditions of epidemic malaria, where risk of infection may be very high, spleen rates may be close to nil because exposure is acute rather than chronic. The spleen rate is useful as a relative measure of risk only where stable malaria prevails. The distinction between hyperendemic and holoendemic on the basis of spleen rate in adults, developed on the basis of observations in sub-Saharan Africa, does not appear to hold true on the island of New Guinea where adults consistently have enlarged spleens in the face of holoendemic malaria.

A secondary estimate of risk utilizing spleen measurements is the average enlarged spleen (AES). The AES represents the mean Hackett score derived from a sample of enlarged spleens. Table 2 describes grading of spleen enlargement on the basis of palpation.

Spleens with Hackett scores of zero are not included in the calculation of AES. A higher AES between two sites with comparable spleen rates may be interpreted as consistent with higher risk. A recent malariometric survey in Papua New Guinea found that, although spleen rates did not vary with altitude for children less than $10 \mathrm{yr}$ of age, AES decreased with increasing altitude, closely paralleling altitude specific prevalence of malaria (4). 


\subsubsection{Serology}

Serological tests have been applied to demonstrate exposure to infection among individual subjects. Unfortunately, no serological test reliably assesses either degree of naturally acquired immunity or the extent of exposure in individuals. Risk of exposure among groups, however, may be assessed by serological analyses. Detection of antibodies to the circumsporozoite protein (CSP) in European travelers returning from malarious areas suggests that anti-CSP antibodies can serve as an indicator of the relative risk of infection in travelers to specific regions (5-7). The prevalence of antibodies to malaria antigens such as CSP, merozoite surface antigen (MSA), and erythrocytic stage antigens has also served as an indirect indicator of risk of infection $(2,8)$ and in some studies correlates with spleen rates $(\boldsymbol{8})$, prevalence of positive smears $(\mathbf{1}, \boldsymbol{8})$, and parasite density (2). Serological assays may also be useful in assessing risk in regions with recent increases in transmission during epidemics or with declining transmission during eradication efforts (9-11). However, no currently available serological assay system can reliably serve as a quantitative measurement of exposure.

\section{Direct Estimates of Risk of Malaria}

\subsection{Passive Surveillance}

\subsubsection{Passive Case Detection}

Passive case detection (PCD) is the detection and reporting of malaria cases restricted to people seeking treatment for illness at a health post, clinic, or hospital. The PCD is reported as simply the number of cases treated. The PCD proportion, or ratio of malaria cases to some defined total (hospitalizations, febrile illnesses, deaths), is often used to gauge the burden of illness caused by malaria relative to all other causes definable in the setting from which those data were collected. This is often called the PCD rate, although it is actually a proportion. The quantitative value of the PCD rate varies according to the rigor of diagnostic procedures and the likelihood of malaria parasitemia masking some other underlying cause of illness (see Subheading 3.2.8.). The availability of reporting health-care delivery facilities also impacts interpretation of PCD data. PCD data are used to gauge risk in communities with the assumption that virtually all infections prompt seeking of treatment at a reporting facility.

In a setting where malaria is reported on the basis of microscopically confirmed infection, and the proportion of fevers caused by malaria is relatively low, the PCD rate may serve as a reliable estimate of risk of malaria relative to other febrile illnesses in the community. In general, this scenario is true where malaria is hypo- to mesoendemic and health-care providers are more likely to report malaria on the basis of diagnostic criteria that exclude other more common causes of febrile illness. In this scenario, the reported PCD proportion may carry good sensitivity and specificity.

The PCD proportion as a measure of risk is less reliable where malaria is hyper- to holoendemic. This problem stems from three important features specific to heavily endemic areas. First, because malaria dominates as a cause of febrile illness, healthcare providers tend to make presumptive diagnoses of malaria in patients presenting with fever. Thus, other causes of febrile illness are often erroneously classified as malaria so that the specificity of the PCD proportion may be extremely low. Second, because self-treatment often relieves symptoms, the true burden of malaria in the com- 
munity may be underreported. Finally, naturally acquired immunity in hyper- to holoendemic regions leaves most infections unnoticed at treatment facilities.

The PCD totals may be analytically applied in a variety of ways. If the PCD total is believed to have captured most infections in the community, then monthly or annual PCD case totals may be divided by the mid-interval population of the areas served by the reporting facilities to define incidence of infection.

Incidence of malaria $=$ malaria $\mathrm{PCD}$ total $/ \mathrm{mid}-$ interval population $/$ unit time

More often, a PCD proportion is applied as a direct measure of the contribution of malaria to febrile disease in communities expressed as a percentage.

$\mathrm{PCD}$ proportion $=($ malaria cases $/$ patients seeking care for febrile illness $) \times 100$

\subsubsection{Annual Parasite Incidence}

The number of malaria cases per 1000 population per year is called the annual parasite incidence, or API. The API represents the most broadly applied measure of risk of infection. Many health authorities rely upon the API as the core measure of risk of infection. The statistic is often used as the basis for comparing risk between communities, districts, provinces, and nations. The means of deriving the numerator for the API, "cases of malaria", varies a great deal. Comparisons of risk based on the API demand consideration of the sources and case definitions; for example, clinical diagnosis versus smear-confirmed diagnosis for reported total cases. The API numerator often represents a hybrid of PCD and active surveillance methods (see below), and the relative contributions of each impact interpretation of the API.

Annual parasite incidence $=$ reported infections $/ 1000$ person $($ mid-year $) /$ year

A statistic used to help interpret the API is the annual blood examination rate, or ABER. This is the number of blood films examined per 1000 population. The ABER reflects the degree of diagnostic effort made to identify malaria. For example, between two locations having comparable API estimates, the location having the lower ABER may be considered higher risk because less effort produced an equal density of infections.

Annual blood examination rate $=$ blood film exams/1000 person $($ mid-year)/year

\subsection{Active Surveillance}

\subsubsection{Active Case Detection}

Systematic screening of communities for people with fever and examination of blood films collected from them is called active case detection (ACD). The analytical application of infections discovered by ACD varies. The data collected by ACD may be applied as follows:

Fever rate $=($ people with fever/people examined for fever $) \times 100$

Malaria among fevers rate $=($ people with malaria/people with fever $) \times 100$

These estimates serve as measures of the prevalence of fever in the community at sampling and the proportion of fevers likely caused by malaria, respectively. Health officers often use ACD to monitor the progress of control efforts within specific areas, 
but also include these detected cases into the numerator for API. Thus, the vigor of case detection impacts on the API. In this sense, the importance of interpreting the API in the context of ABER may be appreciated.

\subsubsection{Active Case Survey}

The primary distinction between active case survey (ACS) and ACD is sampling that does not exclude people without fever. Whereas ACD estimates the prevalence of fever and the proportion of fevers caused by malaria, ACS measures the prevalence of parasitemia independently of fever. The ACS may be the best approach to assessing risk of infection in communities where parasitemia often occurs without fever, that is, where malaria is hyper- to holoendemic. The survey includes recording both febrile and afebrile (or apyrexic) malaria cases. The analytical application of ACS may include the following:

Point prevalence of malaria $=($ people with parasitemia $/$ people examined $) \times 100$

Febrile malaria rate $=($ people with febrile malaria/people with malaria $) \times 100$

Point prevalence refers to the ratio of parasitemic individuals to the total number of individuals examined at a single point in time. This provides an estimate of risk by indicating the number of people infected at any given time with higher prevalence indicating higher rates of transmission. However, for diseases like malaria that may be seasonal in some locations, the point prevalence in June might not be a good estimator of risk for an individual traveling to the area in November.

\subsubsection{Gametocyte Rate}

Analysis of point prevalence data often includes specific designation of the prevalence of gametocytemia in study subjects, the carriage of sexual stage parasites that can be transferred to feeding mosquitoes to complete the life cycle and allow new infections of human hosts. In highly endemic areas where immunity to disease often develops, such individuals are frequently asymptomatic and remain undiagnosed and untreated, serving as reservoirs for new infection in the community. Because gametocytemia declines with increasing age in malaria endemic regions $(\mathbf{1 , 1 2})$, gametocyte rates in young children may serve as an indicator of risk, particularly when comparing gametocyte rates between two different locations.

\subsubsection{Period Prevalence}

Compared to point prevalence, period prevalence may better reflect risk for persons who will be exposed to infection over an extended period. This may be especially true where risk fluctuates appreciably. Whereas point prevalence addresses the question of whether a person currently has malaria, period prevalence addresses the question of whether that subject had malaria at any time during the period under investigation. This serves as a measure of the probability that an individual in a defined population will be a case at any given time over a defined period. The numerator of this estimate includes both newly incident cases (see below) and cases that may have developed before the survey was initiated. The time of onset of infection may not be known, especially in endemic regions where some subjects may be asymptomatic and remain undiagnosed. Thus, incident cases and prevalence may not be distinguishable. Assuming a stable population over time, period prevalence is calculated as follows: 
Period prevalence $=($ people with malaria at start of period + new cases of malaria during period)/population under study) $\times 100$

Period prevalence requires identifying a sample of individuals from a population, screening each of them for malaria on enrollment, and systematically repeating malaria smears during the defined period in order to identify new cases. Multiple infections among any given individual allows the possibility of a period prevalence of $>100 \%$. The greatest limitation with period prevalence is the fact that many population totals are dynamic. The migration of people, and other important changes like mass drug administration, chemoprophylaxis, or any other intervention that alters the number of people at risk, makes the drawing of meaningful statistical inference from measures of period prevalence difficult. In many instances, the effort required to define period prevalence would be better spent measuring incidence, which provides much less ambiguous measures of risk (see Subheading 3.3.).

\subsubsection{Cumulative Incidence}

Cumulative incidence $(\mathrm{CI})$ represents the probability or proportion having malaria or a specific outcome of infection, for example, cerebral malaria, relapse, death, or chemotherapeutic failure over defined intervals. Cumulative incidence is often referred to as an "attack rate," even though the estimate represents a proportion rather than a true rate. The measurement of CI requires a prospective study of people free of infection at the outset. These may be uninfected people newly arriving in a malarious area or people cured of malaria immediately before the observation period. The number of new infections is divided by the number of people at risk, expressed in the context of the period of observation. For example, if one follows 100 people for 10 weeks and 25 get malaria, then the 10 -week cumulative incidence of malaria is $25 \%$. More often, larger populations are followed for longer periods, and the estimate may be complicated by losses to follow-up, migration, or death by other causes.

When individual follow-up times vary for study subjects, a convenient means of calculating cumulative incidence is the actuarial method (also called life table). This approach takes into account the loss of individuals from the study population in the denominator by presuming that the mean withdrawal time occurred at the midpoint of the surveillance interval. For example, in a 1-yr study of malaria with monthly intervals of observation, all subjects lost to follow-up would be assumed to have done so at the midinterval, that is, $2 \mathrm{wk}$. This approach conveniently averages person-time losses across the interval. This is especially useful when such losses are likely, for example, the occurrence of vivax malaria among subjects recruited to gauge the incidence density of falciparum malaria. The actuarial method for estimating CI is as follows:

Cumulative incidence $=$ attack rate $(\%)$ over a defined period

Cumulative incidence $($ actuarial $)=$ incident cases/[population at start of study $-($ number of withdrawals/2)]

\subsubsection{Incidence Density}

Incidence density of malaria estimates the risk of infection in a population expressed as a true rate; that is, the number of new infections per unit person- time. This estimate requires a cohort of people who are free of infection and prospectively followed over a 
defined period. This is most often accomplished by giving radical curative therapy before the follow-up phase, but newly arrived migrants into malarious areas may also provide a suitable cohort without radical cure. Incidence density is calculated simply as the number of new infections divided by the sum of person-time at risk. People lost to follow-up contribute person-time to the denominator up to the point of loss. Their contribution should not be counted for the full period of the study. For example, if one follows 100 subjects for $52 \mathrm{wk}$ and 25 become infected, the incidence density is 25 infections per 100 person-years, or 0.25 infections per person-year, assuming each individual contributed $52 \mathrm{wk}$ of follow-up time. This overly simplistic example assumes no losses to follow-up. However, a subject infected at wk 2 contributes only 2 wk of person-time to the denominator, not 52. Assume that infections occur evenly over the $52 \mathrm{wk}$ and that approximately one infection occurs every $2 \mathrm{wk}$. In this scenario, losses in person-time at risk due to infection outcomes amount to 650 person-weeks, or $12.5 \mathrm{yr}$. Taking these losses into account, the incidence density would be estimated at 25 infections/87.5 person-years, or 0.29 infections/person-year. Further, assume that 20 people were lost follow-up at anywhere from wk 1 to wk 51 of the observation period, yielding a total loss of 12.5 person-years at risk. Thus, the true incidence density in the hypothetical cohort would be estimated as 25 infections per 75 personyears, or 0.33 infections/person-year (or everyone experiencing, on average, an infection once every $3 \mathrm{yr}$ ).

Incidence density = infections/person-year at risk

\subsubsection{Attributable Risk}

Attributable risk (or risk difference) represents an estimate of the risk of disease that may be attributed to a specific exposure. In its simplest form, it is the additional amount of disease in those exposed over the background amount of disease in the unexposed population and is given by:

$$
A R=I_{\mathrm{e}}-I_{\mathrm{u}}
$$

where $I_{\mathrm{e}}$ is the incidence in the exposed population and $I_{\mathrm{u}}$ is the incidence in the unexposed population.

In conducting malaria studies in transmission areas, it is difficult to reliably differentiate between reinfection and recurrent parasitemia following therapy. The attributable risk statistic, calculated by subtracting the coincident incidence rate for a given population from the rate of recurrent parasitemia, estimates the rate of therapeutic failure. The efficacy of standard mefloquine therapy against uncomplicated Plasmodium falciparum infections was evaluated in children aged 6 to 24 mo in the KassenaNankana District of northern Ghana, West Africa. The incidence of late recrudescence, or therapeutic failure, was calculated as the difference between the incidence of recurrent parasitemia during wk 3 and 4 after mefloquine therapy and the known attack rate of malaria in the region for the cohort. The incidence of recurrent parasitemia at $\mathrm{d} 28$ was 6.3 infections/person-year at risk. However, this incidence rate approximated the known reinfection rate in this cohort (5.7 infections/person-year). Thus, the observed parasitemia in the treatment group could be almost wholly attributed to the measured reinfection rate in this cohort. This method has been reported previously in comparing the efficacy of antimalarial drug regimens $(\mathbf{1 3}, 14)$. 


\subsubsection{Attributable Fraction}

Attributable fraction represents an estimate of the risk of disease in a community that is attributable to a particular risk factor. Because many symptoms of malaria are non-specific, attributable fraction is a useful measure in looking at clinical markers or case definitions for malaria in a community. The statistic assigns a probability of a single fever episode being due to malaria. For a single exposure variable, the overall attributable fraction is given by

$$
A F=p(R-1) / R
$$

where $p$ is the exposure prevalence among cases and $R$ is the relative risk of disease associated with the exposure (15). The probability that any individual case is attributable to malaria is calculated without multiplying by $p(\mathbf{1 6})$.

Probabilities derived from logistic regression models can increase the precision of the attributable fraction estimates by allowing fever risk as a continuous function of parasite density (16) and can be further extended to include other covariates (17).

Schellenberg et al. (18) used the fraction of fever cases in a population attributable to malaria at each level of parasite density to evaluate the sensitivity and specificity of alternative case definitions for malaria, and to provide a direct estimate of malariaattributable fever. The attributable fraction statistic also has been used to determine the specificity and sensitivity of case-definition thresholds (19).

\section{References}

1. Genton, B., al-Yaman, F., Beck, H. P., Hii, J., Mellor, S., Narara, A., et al. (1995) The epidemiology of malaria in the Wosera area, East Sepik Province, Papua New Guinea, in preparation for vaccine trials. I. Malariometric indices and immunity. Ann. Trop. Med. Parasitol. 89, 359-376.

2. May J., Mockenhaupt, F. P., Ademowo, O. G., Falusi, A. G., Olumese, P. E., Bienzle, U., and Meyer, C. G. (1999) High rate of mixed and subpatent malaria infections in Southwest Nigeria. Am. J. Trop. Med. Hyg. 61, 339-343.

3. Pribadi, W., Sutanto, I., Atmosoedjono, S., Rasidi, R., Surya, L. K., and Susanto, L. (1998) Malaria situation in several villages around Timika South Central Irian Jaya, Indonesia. Southeast Asian J. Trop. Med. Public Health 29, 228-235.

4. Hii, J., Dyke, T., Dagoro, H., and Sanders, R. C. (1997) Health impact assessments of malaria and Ross River virus infection in the Southern Highlands Province of Papua New Guinea. P.N.G. Med. J. 40, 14-25.

5. Nothdurft, H. D., Jelinek, T, Bluml, A., von Sonnenburg, F., and Loscher, T. (1999) Seroconversion to circumsporozoite antigen of Plasmodium falciparum demonstrates a high risk of malaria transmission in travelers to East Africa. Clin. Infect. Dis. 28, 641,642.

6. Jelinek, T., Bluml, A., Loscher, T., and Northdurft, H. D. (1998) Assessing the incidence of infection with Plasmodium falciparum among international travelers. Am. J. Trop. Med. Hyg. 59, 35-37.

7. Cobelens, F. G., Verhave, J. P., Leentvaar-Kuijpers, A., and Kager, P. A. (1998) Testing for anticircumsporozoite and anti-blood-stage antibodies for epidemiologic assessment of Plasmodium falciparum infection in travelers. Am. J. Trop. Med. Hyg. 58, 75-80.

8. Al-Yaman, F., Genton, B., Kramer, K.J., Taraika, J., Chang, S. P., Hui, G. S., and Alpers, M. P. (1995) Acquired antibody levels to Plasmodium falciparum merozoite surface antigen 1 in residents of a highly endemic area of Papua New Guinea. Trans. R. Soc. Trop. Med. Hyg. 89, 555-559.

9. Bruce-Chwatt, L. J., Draper, C. C., and Konfortion, P. (1973) Seroepidemiologic evidence of eradication of malaria from Mauritius. Lancet 2, 547-551.

10. Lobel, H. O., Najera, A. J., Ch'en, W. I., Munore, P., and Mathews, H. M. (1976) Seroepidemiologic investigations of malaria in Guyana. J. Trop. Med. Hyg. 79, 275-284.

11. Tikasingh, E., Edwards, C., Hamilton, P. J. S., Commissiong, L. M., and Draper, C. C. (1980) A malaria outbreak due to Plasmodium malariae on the island of Grenada. Am. J. Trop. Med. Hyg. 29, 715-719.

12. Gilles, H. M. (1993) Epidemiology of malaria, in Bruce-Chwatt's Essential Malariology, 3rd ed., Arnold, London.

13. ter Kuile, F. O., Dolan, G., Nosten, F., Edstein, M. D., Luxemburger, C., Phaipun, L., Chongsuphajaisiddhi, T., Webster, H. K., and White, N. J. (1993) Halofantrine versus mefloquine in treatment of multidrug-resistant falciparum malaria. Lancet. 341, 1044-1049. 
14. von Seidlein, L., Milligan, P., Pinder, M., Bojang, K., Anyalebechi, C., Gosling, R., et al. (2000) Efficacy of artesunate plus pyrimethamine-sulphadoxine for uncomplicated malaria in Gambian children: a double-blind, randomised, controlled trial. Lancet 355, 352-357.

15. Rothman, K. J., Adami, H. O., and Trichopoulos D. (1998) Should the mission of epidemiology include the eradication of poverty? Lancet 352, 810-813.

16. Smith, T., Genton, B., Baea, K., Gibson, N., Taime, J., Narara, A., et al. (1994) Relationships between Plasmodium falciparum infection and morbidity in a highly endemic area. Parasitology 109, 539-549.

17. Prybylski, D., Khaliq, A., Fox, E., Sarwari, A. R., and Strickland, G. T. (1999) Parasite density and malaria morbidity in the Pakistani Punjab. Am. J.Trop. Med. Hyg. 61, 791-801.

18. Smith, T., Schellenberg, J. A., and Hayes, R. (1994) Attributable fraction estimates and case definitions for malaria in endemic areas. Stat. Med. 13, 2345-2358.

19. McGuinness, D., Koram, K., Bennett, S., Wagner, G., Nkrumah, F., and Riley, E. (1998) Clinical case definitions for malaria: clinical malaria associated with very low parasite densities in African infants. Trans. R. Soc. Trop. Med. Hyg. 92, 527-531. 